\title{
Tratamiento de los \\ encondromas de la mano con cerámicas biorreabsorbibles
}

\author{
A. Fuentes Díaz ${ }^{(1)}$, E. Mackenney Carrasco ${ }^{(2)}$ \\ (1) JeFe de Sección. Servicio C.O.T. Hospital Vega Baja. ORihuela (Alicante).

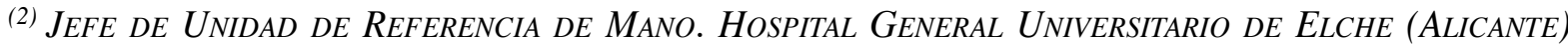

\author{
Correspondencia: \\ Dr. Alfonso Fuentes Díaz \\ Servicio de C.O.T. Hospital Vega Baja \\ Ctra. Orihuela-Almoradí, s/n \\ 03314 San Bartolomé-Orihuela. España \\ Teléfono 965877761 \\ Fax 965877763 \\ e-mail: al.fuentesd@coma.es
}

Objetivos: Aunque el tratamiento estándar de los encondromas es el curetaje más relleno con hueso autólogo, hay autores que recomiendan la utilización de material sintético para evitar morbilidad en la zona dadora. En este sentido presentamos nuestra experiencia de 12 casos tratados con curetaje y relleno con cerámicas biorreabsorbibles.

Material y Método: Nueve encondromas estaban localizados en las falanges y 3 en los metacarpianos. Tras su curetaje, 10 se rellenaron con hidroxiapatita de origen natural, 1 con fosfato tricálcico y otro con sulfato cálcico.

Resultados: Tras un seguimiento medio de 26,3 meses, no se encontraron complicaciones postquirúrgicas, fracturas ni recidivas. Se objetivó la incorporación de la cerámica en todos los casos aunque sólo en uno de ellos se produjo su reabsorción completa.

Conclusiones: Las ventajas de los materiales sintéticos son: disponibilidad ilimitada, disminución de la duración de la intervención y ausencia de complicaciones en la zona donante. No obstante el resultado final va a depender más de la técnica quirúrgica que del material de relleno, ya que un curetaje exhaustivo y una reposición de la ventana cortical son fundamentales a la hora de evitar recidivas y prevenir complicaciones.

Palabras clave: encondroma, mano, tratamiento, cerámicas.
Aim: The standard treatment of enchondromata is by curettage of the tumour and filling the resultant cavity with cancellous bone graft. To avoid donorsite morbility, treatment without grafting or packing the defect with bone substitutes has been suggested. The purpose of this report is to present the clinical and radiological outcome of 12 cases treated by curettage and implantation of a biodegradable ceramic.

Material and Method: Nine of the enchondromata were in the phalanx and 3 in the metacarpal bone. After curettage 10 cases were filled with biologic hidroxyapatite, one case with tricalcium phosphate and other case with calcium sulphate.

Results: At a mean follow-up of 26,3 months, non postsurgical complications, fractures or local recurrences were observed. Bony incorporation was uneventful in all cases although only a case showed complete absorption.

Conclusion: The advantages of synthetics materials are: unlimited availability, the operative time is shortened and complications at the cancellous bone donor site are avoided. However for the final result is more important an accurate surgical technique that the grafting material employed: meticulous removing of the tumour tissue, replacement of the cortical window and preservation of the periostium are fundamental to prevent local recurrences and complications.

Key words: enchondroma, hand, surgery, ceramics. 


\section{INTRODUCCIÓN}

L os encondromas son los tumores óseos primarios más frecuentes de la mano ${ }^{1-7}$. Tienen predilección por los radios cubitales y las falanges proximales ${ }^{3,6,8}$. Su incidencia es desconocida ya que muchos son asintomáticos y sólo se diagnostican al hacer una radiografía por otro motivo ${ }^{7}$. Otros son identificados al ocasionar una fractura patológica o producir una tumefacción más o menos dolorosa, lo que conduce a su diagnóstico ${ }^{3,5,9}$. El tratamiento estándar es el curetaje más relleno con hueso autólogo, habitualmente hueso esponjoso obtenido de la cresta iliaca. ${ }^{10}$ Con ello se consigue obtener un diagnóstico anatomopatológico, resecar el tumor, prevenir la recurrencia y la producción de una fractura patológica ${ }^{1,2,4,5,7,9}$. No obstante esta pauta de tratamiento no está exenta de inconvenientes, como la necesidad de otro tipo de anestesia (incluyendo la general), el aumento en la duración de la intervención y la hospitalización, así como las complicaciones en la zona dadora (dolor prolongado, hematoma, infección, lesión del nervio femorocutáneo, etc.) que según algunas series se cifra hasta en un $8,6 \% 1,2,9,11$.

El objetivo de este trabajo es valorar el resultado del tratamiento de los encondromas de la mano con curetaje y relleno con sustitutos óseos sintéticos, en concreto cerámicas biorreabsorbibles.

\section{MATERIAL Y MÉTODO}

Entre noviembre de 1999 y abril de 2007 se trataron 12 pacientes con encondromas en la mano (6 por cada uno de los autores de este trabajo). Se trataba de 7 hombres y 5 mujeres de edades comprendidas entre 13 y 69 años (Tabla I).

Nueve tumores afectaban a las falanges y 3 a los metacarpianos, siendo el quinto radio el más comúnmente afectado (5 ocasiones). Los encondromas se clasificaron según la modificación que realizaron Hasselgren et al. ${ }^{5}$ de la clasificación propuesta por Takigawa ${ }^{8}$. En ella se

\section{Tabla I - CARACterísticas DE LA SERIE}

\begin{tabular}{|ccccccccc|}
\hline Caso & $\begin{array}{c}\text { Género } \\
\text { (H/M) }\end{array}$ & $\begin{array}{c}\text { Edad } \\
\text { (años) }\end{array}$ & $\begin{array}{c}\text { Lado } \\
\text { (dominante) }\end{array}$ & Localización & $\begin{array}{c}\text { Motivo } \\
\text { diagnóstico }\end{array}$ & $\begin{array}{c}\text { Demora dx-tto } \\
\text { (meses }\end{array}$ & $\begin{array}{c}\text { Material } \\
\text { relleno }\end{array}$ & $\begin{array}{c}\text { Seguimiento } \\
\text { (meses) }\end{array}$ \\
\hline 1 & H & 26 & (D) & M1 & Casual & 4 & Prosteon & 48 \\
\hline 2 & M & 34 & D & F2 D4 & Tumefacción & 6 & Prosteon & 48 \\
\hline 3 & H & 58 & (D) & M5 & Fractura & 60 & Prosteon & 36 \\
\hline 4 & H & 44 & (D) & F1 D5 & Casual & 13 & Calciresorb & 21 \\
\hline 5 & M & 48 & D & M3 & Casual & 12 & Prosteon & 23 \\
\hline 6 & M & 13 & (I) & F1 D5 & Fractura & 18 & Prosteon & 24 \\
\hline 7 & H & 17 & (D) & F1 D2 & Casual & 6 & Osteoset & 12 \\
\hline 8 & H & 22 & (D) & F1 D1 & Casual & 6 & Prosteon & 23 \\
\hline 9 & H & 38 & I & F2 D3 & Casual & 6 & Prosteon & 21 \\
\hline 10 & H & 49 & I & F2 D3 & Tumefacción & 4 & Prosteon & 20 \\
\hline 11 & M & 49 & I & F1 D5 & Tumefacción & 4 & Prosteon & 20 \\
\hline 12 & M & 69 & I & F2 D5 & Tumefacción & 6 & Prosteon & 20 \\
\hline Media & & 38,5 & & & & 12 & & 26,3 \\
\hline
\end{tabular}




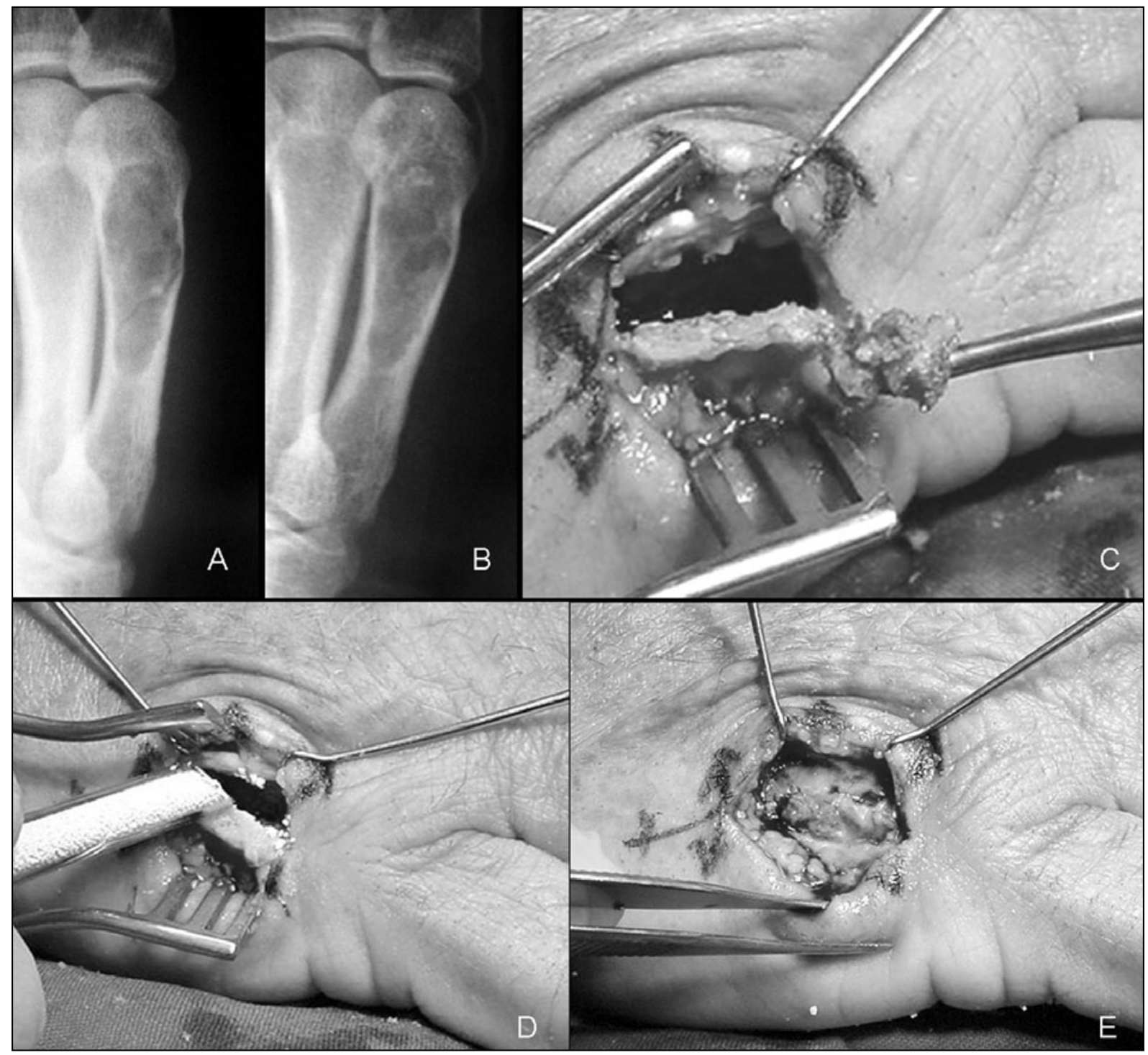

Figura 1. Caso $n^{o}$ 3. A. Encondroma de M5 diagnosticado al ocasionar una fractura patológica. Con tratamiento conservador ésta consolidó en pocas semanas, pero el encondroma no se resolvió. B. Radiografía 5 años más tarde donde todavía se aprecia la lesión en M5, por lo que se decidió el tratamiento quirúrgico. C. Por vía dorsal, se realizó una ventana ósea para el curetaje completo de la lesión. D. Se rellenó la cavidad con gránulos de hidroxiapatita. E. Se repuso la ventana cortical y se suturó el periostio.

valoran 4 parámetros: la distribución (monostótica o poliostótica), el tipo (monocéntrico, policéntrico o gigante), la localización (central, excéntrica o asociada) y la forma (expansiva, o no expansiva). Todos nuestros casos eran monostóticos, la mayoría de ellos monocéntricos y centrales, y el 50\% expansivos (Tabla II). En la mitad de los casos el motivo del diagnóstico fue el hallazgo casual, la fractura patológica en 2 de ellos y una tumefacción dolorosa de la mano en los 4 casos restantes. El tiempo me- dio entre el diagnóstico y el tratamiento fue de 12 meses.

Las intervenciones se realizaron mediante anestesia regional, con exanguinación del miembro intervenido y manguito neumático a nivel del brazo y en régimen ambulatorio. En todos ellos se realizó por un abordaje dorsal una ventana ósea de tamaño suficiente para permitir el curetaje de la lesión, y el material obtenido se remitió para la confirmación anatomopatológica del diagnóstico (Figura 1C). Diez casos se 


\begin{tabular}{|c|c|c|c|c|}
\hline \multicolumn{5}{|c|}{$\begin{array}{l}\text { Tabla II - Clasificación de TAKIGAWA }{ }^{8} \text { MOdificadA } \\
\text { POR HASSELGREN ET AL. }{ }^{5} \text { DE NUESTROS } \\
\text { CASOS }\end{array}$} \\
\hline Caso & $\begin{array}{l}\text { Distribución } \\
\text { (M/P) }\end{array}$ & $\begin{array}{l}\text { Tipo } \\
\text { (m/p/g) }\end{array}$ & $\begin{array}{l}\text { Localización } \\
\text { (c/e/a) }\end{array}$ & $\begin{array}{l}\text { Forma } \\
\text { (E/NE) }\end{array}$ \\
\hline 1 & $M$ & $p$ & c & NE \\
\hline 2 & $M$ & $g$ & c & E \\
\hline 3 & $M$ & $\mathrm{~m}$ & c & $E$ \\
\hline 4 & $M$ & g & c & $E$ \\
\hline 5 & M & $\mathrm{m}$ & c & $E$ \\
\hline 6 & $M$ & $\mathrm{~m}$ & e & NE \\
\hline 7 & $M$ & $\mathrm{~m}$ & c & NE \\
\hline 8 & M & $\mathrm{p}$ & c & NE \\
\hline 9 & $M$ & $\mathrm{~m}$ & e & NE \\
\hline 10 & $M$ & $\mathrm{~m}$ & e & $E$ \\
\hline 11 & $M$ & $\mathrm{~m}$ & e & $E$ \\
\hline 12 & $M$ & $\mathrm{p}$ & c & NE \\
\hline Total & $12 \mathrm{M}$ & $7 \mathrm{~m} / 3 \mathrm{p} / 2 \mathrm{~g}$ & $8 c / 4 e$ & $6 \mathrm{E} / 6 \mathrm{NE}$ \\
\hline
\end{tabular}

$\mathrm{M}=$ monostótico, $\mathrm{P}=$ poliostótico; $\mathrm{m}=$ monocéntrico, $\mathrm{p}=$ policéntrico, $\mathrm{g}=$ gigante; $\mathrm{C}=$ central, e=excéntrico, $\mathrm{a}=$ asociado; $\mathrm{E}=$ expansiva, $\mathrm{NE}=\mathrm{no}$ expansiva

rellenaron con Pro Osteon TM 200 (Interpore Cross International) que es una hidroxiapatita de origen natural, uno con Calciresorb (Ceraver Osteal) que es un compuesto de fosfato tricálcico y otro con Osteo-Set (Wright Medical Technology) que es un sulfato cálcico; todos se introdujeron en forma de gránulos de 0,5-1 mm, o bolitas de $3 \mathrm{~mm}$ de tamaño, mediante el uso de jeringas precargadas (Figura 1D). Tras ello se repuso la ventana ósea y se suturó el periostio (Figura 1E). A nueve pacientes se les permitió iniciar la movilidad completa de la mano y a 3 se les colocó una inmovilización adicional durante 2 semanas como protección de la reparación del aparato extensor digital. Al final del seguimiento se realizó la valoración de los pacientes siguiendo los 4 criterios propuestos por Takigawa ${ }^{8}$ : apariencia (aceptable, o no aceptable), rango de movilidad activa (mayor o me- nor al $80 \%$ de la contralateral), fuerza de prensión (puño) y pulpejo-pulpejo con dinamómetros Jamar y B\&L respectivamente (mayor, o menor, al $80 \%$ de la contralateral) y evidencia radiológica de curación sin acortamiento, deformidad, artrosis o recurrencia tumoral. Los resultados se consideraron excelentes si cumplían los 4 criterios, buenos si sólo cumplían 3, regulares si sólo 2 y malos si no cumplían ninguno o sólo uno.

\section{RESULTADOS}

En todos los casos el estudio anatomopatológico confirmó el diagnóstico de encondroma. Tras la cirugía no hubo lesiones vasculonerviosas, tendinosas, infecciones superficiales o profundas ni fracturas por sobrecarga. Todos los 


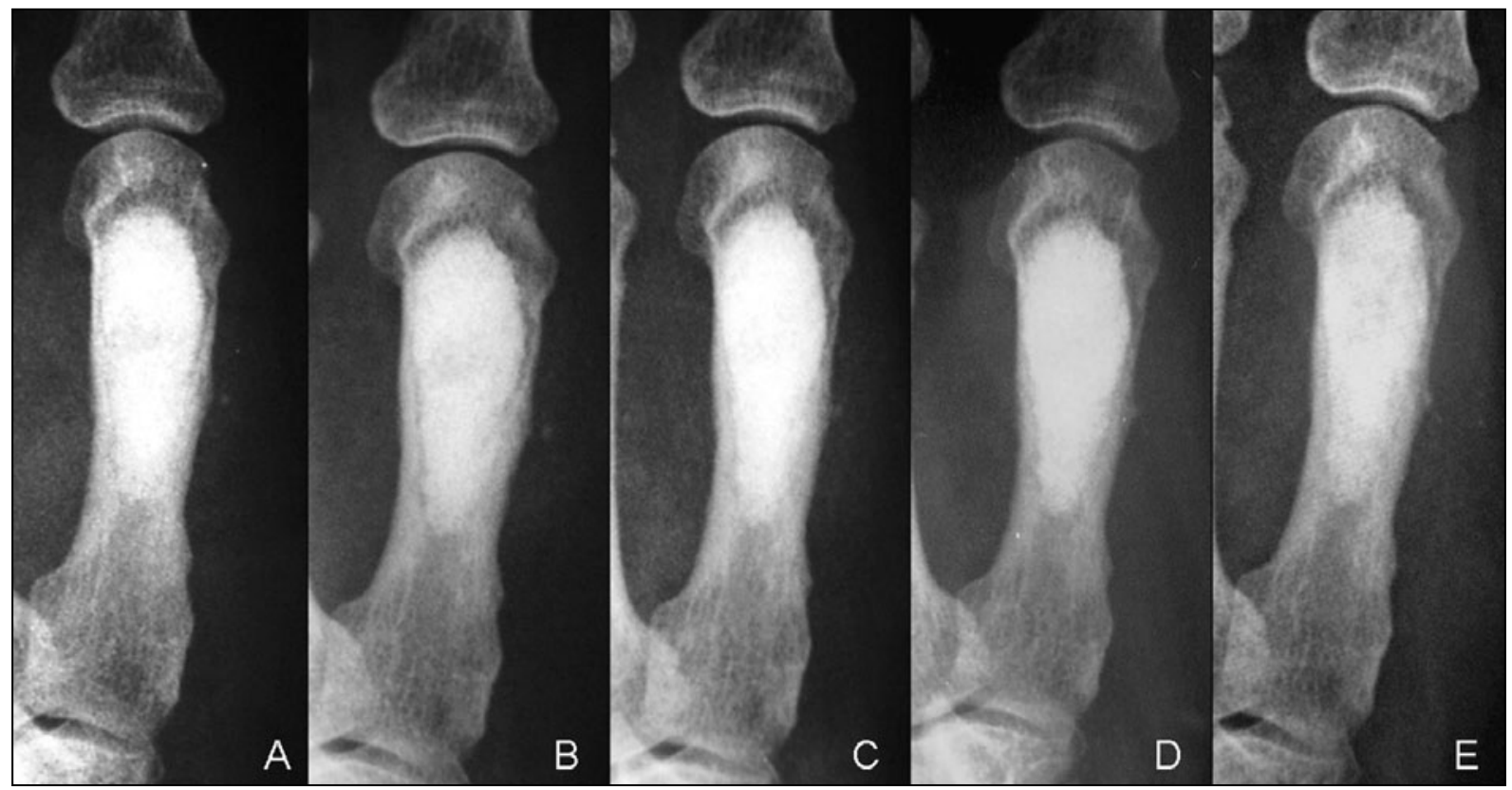

Figura 2. Caso $n^{o}$ 3. Evolución radiográfica. La cerámica de HA es más radiopaca que el hueso receptor, y ambos están separados por una fina línea radiolucente perilesional. Con el paso del tiempo se aprecia una leve reabsorción de la HA: sus márgenes se van haciendo más irregulares, la línea radiolucente se va obliterando y se observa un ligero difuminado en su interior. A. Control postoperatorio. B. A los 2 meses. C. Control a los 6 meses. D. Rx al año. E. Aspecto los 3 años.

pacientes recobraron su estatus funcional prequirúrgico entre las 4-6 semanas de la intervención. El seguimiento medio fue de 26,3 meses (rango: 12-48). Todos los resultados fueron catalogados como excelentes y ningún paciente tuvo recurrencia del tumor, tumefacción residual o deformidad. Se constató incorporación del material de relleno en todos los casos, aunque sólo en uno se produjo su reabsorción completa (caso $n^{\circ} 7$ ). No se encontraron diferencias en los resultados en relación al hueso afectado, al tiempo de evolución de la tumoración o con alguno de los parámetros valorados en la clasificación utilizada.

\section{DISCUSIÓN}

El sustituto óseo ideal debe ser: osteogénico, biocompatible, bioabsorbible, capaz de proporcionar soporte estructural, que se incorpore y reemplace fácilmente por hueso, clínicamente fácil de usar, de disponibilidad ilimitada, con bajo riesgo de infección, mejor si es radiopaco y con una buena relación coste-beneficio ${ }^{12-15}$.
El hueso autólogo y los sustitutos óseos pueden tener propiedades osteogénicas, osteoinductivas y osteoconductivas. Recordemos los siguientes conceptos: osteogénesis (formación de hueso nuevo en el sitio receptor), osteoinducción (estimulación de la células precursoras óseas del huésped) y osteoconducción (propiedad de un material para permitir en su interior el crecimiento de células osteoprogenitoras y la formación ósea $)^{16,17}$. El único material que posee estas 3 propiedades es el hueso esponjoso autólogo, todos los demás sustitutos óseos incluidas las cerámicas, la matriz ósea desmineralizada, las proteínas óseas morfogenéticas, el plasma rico en plaquetas etc., sólo poseen una o dos de estas propiedades ${ }^{13,18}$.

Entre los derivados óseos sintéticos se encuentran las cerámicas, las cuales se dividen en varios grupos: las hidroxiapatitas (HA) de origen natural bien derivadas del coral o de hueso bovino, las HA sintéticas, el fosfato tricálcico (TCP), el sulfato cálcico, el fosfato cálcico bifásico (DCP) que es un derivado de los 2 anteriores, y por último los cementos de fosfato cálcico (CPC). Todas las cerámicas son sólo osteo- 


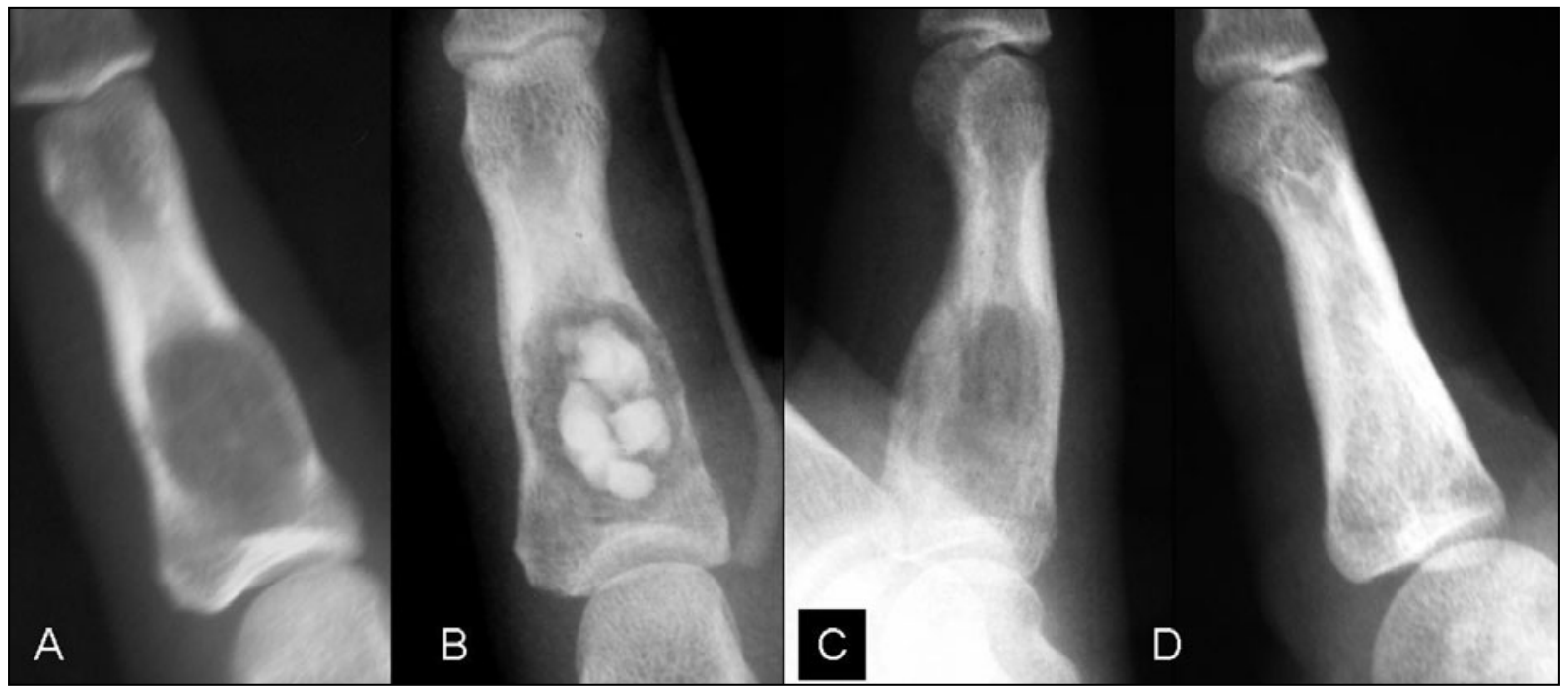

Figura 3. Caso $n^{o}$ 7. Evolución radiográfica del único caso en que la reabsorción de la cerámica fue completa. A. Encondroma $F 12^{o}$ dedo mano derecha. B. Control postoperatorio tras curetaje y relleno con sulfato cálcico. C. Control a los 2 meses. D. Aspecto al año de la intervención.

conductivas, es decir sólo sirven de andamiaje para que las células osteoformadoras produzcan hueso en su interior y se comercializan en una amplia gama de presentaciones: gránulos y bloques de diferentes tamaños, polvos, geles, masillas, pastas inyectables, tiras etc. ${ }^{12,13,15-17,19}$.

Se considera que un sustituto óseo se ha incorporado al hueso cuando la transición entre ambos se sucede sin fisuras y no existe una hendidura entre ellos. Para que ocurra tiene que producirse una reabsorción parcial o completa de este material y su sustitución por hueso neoformado $^{14,16,17}$. Por este motivo todos estos materiales además de ser bioactivos, deben ser reabsorbibles ${ }^{13,14}$. Todas las cerámicas se biodegradan y reabsorben en mayor o menor medida al contacto con los fluidos biológicos, dependiendo de varios factores, algunos inherentes a las propiedades específicas del material (tamaño de la partícula, porosidad, estructura o forma de presentación ${ }^{19}$, y otros que dependen de la localización y del tamaño de la lesión en la que se utilicen, del ambiente en el lecho receptor o de las condiciones propias del paciente (edad, estado general, etc. $)^{17}$. La velocidad de reabsorción es mayor para el sulfato cálcico (3060 días) que para el TCP (6 meses) o la matriz ósea desmineralizada (7 años). La reabsorción de las HA biológicas es lenta e impredecible y a las HA sintéticas se las considera prácticamente como no reabsorbibles ${ }^{11-13,15,19,20}$. La del CPC es también muy variable, depende del preparado en concreto del que se trate, aunque por regla general la falta de porosidad los hace muy estables en el tiempo y difícilmente reabsorbibles ${ }^{2,15,} 20$.

En el periodo postoperatorio inicial las cerámicas son más radiopacas que el hueso receptor, y poseen un reborde radiolucente bien definido que las delimita de éste. ${ }^{16,17}$ Con el tiempo constatamos una obliteración de este reborde perilesional con una irregularidad en los bordes de la cerámica y un difuminado en su interior, todo ello indicativo de incorporación ósea, aunque la reabsorción de la HA nunca fue completa ni siquiera en los casos con un seguimiento más largo (Casos 1, 2 y 3) (Figura 2). Sin embargo, la reabsorción en el único caso que utilizamos sulfato cálcico fue completa al año (Figura 3).

Las referencias bibliográficas del tratamiento de encondromas de la mano con cerámicas biorreabsorbibles no es muy numerosa, las series son de pocos pacientes, con escaso seguimiento a largo plazo y en cada una de ellas se emplea un biomaterial distinto. Wulle ${ }^{10}$ y Gaasbeek et al. ${ }^{11}$ utilizan el Gypsum o Yeso de Paris (sulfato cálcico dihídrico/fosfato cálcico) en 
4 y 19 casos, y a 14 y 53 meses respectivamente de seguimiento medio. Baer et al. ${ }^{21}$ emplean Endobon (Merck Biomaterials), una HA biológica de origen bovino en 22 casos, de los que 16 eran encondromas y con un seguimiento medio de 89 meses. Jacoulet y Fauré ${ }^{1}$ presentan 5 casos con 28 meses de seguimiento utilizando TCP. Y por último Joosten et al. ${ }^{2}$ y Yasuda et al. ${ }^{14}$ utilizan en sus series de 8 y 10 casos, a 12 y 41 meses de seguimiento medio, distintos preparados de CPC. Todos ellos obtienen muy buenos resultados funcionales, con escasas o nulas complicaciones y aunque constatan la incorporación ósea de los biomateriales, su reabsorción es muy diferente: entre 4 y 10 semanas para el Gypsum, aunque con el TCP la trabeculación se puede observar a los 6 meses, no es hasta los 2 años cuando reaparece el canal medular, y con el CPC los cambios radiológicos son muy escasos con poca evidencia de formación ósea en el tiempo. Estos autores defienden el uso del relleno para el tratamiento de los encondromas ya que con él se minimiza el defecto óseo, se asegura la formación ósea y la rápida adquisición de resistencia, cuando la cortical está muy adelgazada se previenen las fracturas patológicas y permite que se realice una movilización precoz $^{1,11,21}$.

Por el contrario existe otra corriente de autores que opinan que con el curetaje simple es suficiente porque la formación de hueso nuevo y la remodelación de la lesión pueden realizarse sin necesidad de injerto de hueso o de otro material. Además las cerámicas son poco resistentes a la torsión o al cizallamiento, la contribución de la esponjosa a la estabilidad ósea es muy pequeña, y depende fundamentalmente de la integridad de la cortical ${ }^{7,21,22}$. Se evita el riesgo de efectos adversos sobre partes blandas y de dificultad de extracción en caso de necesidad (infección, recidiva, etc.) que tienen materiales como el Yeso de Paris o el CPC. El gasto económico es también menor, y los resultados son completamente equiparables al de los anteriores $4,5,7,9,10,22$.

Pensamos que para encondromas de pequeño tamaño el curetaje simple puede ser suficiente, reservando el relleno para los casos de mayor tamaño o adelgazamiento importante de la cortical. Las ventajas de los biomateria- les son su disponibilidad ilimitada, la disminución de la duración de la intervención, y que con ellos se evitan complicaciones sobre la zona donante.

No obstante el resultado final va a depender más de la técnica quirúrgica empleada que del material usado para su relleno. El tamaño de la ventana ósea debe adaptarse al tamaño de la lesión y permitir que el curetaje sea exhaustivo $^{1,2,8,14}$, incluso hay autores que para asegurarse de ello lo realizan bajo visión endoscópi$\mathrm{ca}^{7}$. La reposición de la ventana cortical y la preservación del periostio aceleran la recuperación de la continuidad del cortex y la resistencia ósea ${ }^{7,9}$. Ningún sustituto óseo proporciona una estabilidad mecánica inmediata, ni siquiera los cementos de CPC poseen resistencia a la torsión o al cizallamiento ${ }^{20,22}$. Sólo la reconstrucción con polimetilmetacrilato más fijación interna proporciona un soporte mecánico inmediato, que permite la movilización precoz y evita la fractura patológica ${ }^{6}$. Por este motivo y porque se han reportado más complicaciones con el tratamiento precoz de los encondromas, la mayoría de autores aconsejan que una vez producida la fractura, se demore la intervención entre 4 y 6 semanas hasta que exista consolidación de la misma ${ }^{9,14,23}$. En nuestra experiencia y al contrario de lo observado por otros autores, no objetivamos mejoría en la apariencia radiológica ni curación del encondroma en ninguno de los casos que sufrieron fractura patológica $^{8}$ (Figura 1A y B). El seguimiento clínico es insuficiente para diagnosticar las recidivas. Gaulke y Suppelna ${ }^{3}$ encuentran en su serie recurrencias asintomáticas incluso a los 10 años de la intervención. Por este motivo aconsejan realizar radiografías periódicas a intervalos de 2-5 años para diagnosticarlas antes de que ocasionen debilidad ósea o fractura patológica.

Creemos que la aportación de nuestra experiencia puede ser de ayuda en un tema tan controvertido como éste, en el que hay muy pocos estudios clínicos a medio y largo plazo a pesar de los numerosos biomateriales comercializados. El conocimiento y correcta aplicación de los conceptos que hemos expuesto, son fundamentales para un adecuado tratamiento de estas lesiones, prevenir sus recidivas y evitar las complicaciones. 
1. Jacoulet P, Faure P. Treatment of enchondromas of the hand with bone substitute. Preliminary report of five cases. $\mathrm{J}$ Hand Surg Br, 1997; 22: 476-8.

2. Joosten $U$, Joist $A$, Frebel $T$, et al. The use an in situ curing hydroxyapatite cement as an alternative to bone graft following removal of enchondroma of the hand. J Hand Surg Br, 2000; 25: 288-91.

3. Gaulke R, Suppelna G. Solitay enchondroma at the hand. Long-term follow-up study after operative treatment. J Hand Surg Br, 2004; 29: 64-6.

4. Tordai $P$, Hoglund M, Lugnegard $\mathrm{H}$. Is the treatment of enchondroma in the hand by simple curettage a rewarding method? J Hand Surg Br, 1990; 15: $331-4$.

5. Hasselgren $G$, Forssblad $P$, Törnvall $A$. Bone grafting unnecessary in the treatment of enchondromas in the hand. $J$ Hand Surg Am, 1991; 16: 139-42.

6. Bickels J, Wittig JC, Kollender $\mathrm{Y}$, et al. Enchondromas of the hand: treatment with curettage and cemented internal fixation. $J$ Hand Surg Am, 2002; 27: 870-5.

7. Sekiya I, Matsui N, Otsuka T, et al. The treatment of enchondromas in the hand by endos-

\section{BIBLIOGRAFÍA}

copic curettage without bone grafting. J Hand Surg $\mathrm{Br}, 1997$ 22: $230-4$.

8. Takigawa K. Chondroma of the bones of the hand. A review of 110 cases. J Bone Joint Surg Am, 1971; 53: 1591-600.

9. Goto T, Yokokura S, Kawano H et al. Simple curettage without bone grafting for enchondromata of the hand: with special reference to replacement of the cortical window. J Hand Surg $\mathrm{Br}, 2002$; 27: 446-51.

10. Wulle $\mathrm{C}$. On the treatment of enchondroma. J Hand Surg Br, 1990; 15: 320-30.

11. Gaasbeek RDA, Rijnberg WJ, van Loon CJM, et al. No local recurrence of enchondroma after curettage and plaster filling. Arch Orthop Trauma Surg, 2005; 125: 42-5.

12. Tay BKB, Patel VV, Bradford DS. Calcium sulphate and calciumphosphate based bone substitutes. Mimicry of the mineral phase of bone. Orthop Clin N Am, 1999; 30: 615-23.

13. Giannoudis PV, Dinopoulos H, Tsiridis E. Bone substitutes: an update. Injury, 2005; 36: S20-7.

14. Yasuda M, Masada K, Takeuchi E. Treatment of enchondroma of the hand with injectable calcium phosphate bone cement. J Hand Surg Am, 2006; 31: 98-102.
15. De Long WG Jr, Einhorn TA, Koval $\mathrm{K}$, et al. Bone grafts and bone graft substitutes in orthopaedic trauma surgery. Critical analysis. J Bone Joint Surg Am, 2007; 89: 649-58.

16. Beaman FD, Bancroft LW, Peterson JJ, et al. Bone graft materials and synthetic substitutes. Radiol Clin N Am, 2006; 44: 451-61.

17. Beaman FD, Bancroft LW, Peterson JJ, et al. Imaging characteristics of bone graft materials. Radiographics, 2006; 26: 373-83.

18. Sen MK, Miclan T. Autologus iliac crest bone graft: should it still be the gold standart for treating nonunions? Injury, 2007; 38: S75-80.

19. LeGeros RZ. Properties of osteoconductive biomaterials: calcium phosphates. Clin Orthop, 2002; 395: 81-98.

20. Gisep A. Research on ceramic bone substitutes: current status. Injury, 2002; 33: B88-92.

21. Baer W, Schaller P, Carl HD. Spongy hydroxyapatite in hand surgery. A five year follow-up. J Hand Surg Br, 2002; 27: 101-3.

22. Geissler WB. Bone graft substitutes in the upper extremity. Hand Clin, 2006; 22: 329-39.

23. Ablove RH, Moy OJ, Peimer $\mathrm{CA}$, et al. Early versus delayed treatment of enchondroma. Am J Orthop, 2000; 29: 771-2. 